\title{
Skin, hair and nails as pointers to neurocognitive disorders in children: our experience.
}

\author{
S R Chandra ${ }^{1 *}$, Akanksha Suresh ${ }^{2}$, CS Vidhya Annapoorni ${ }^{3}$, Rita Christopher ${ }^{4}$, Asheeb A ${ }^{1}$, Thomas Gregor Issac ${ }^{5}$ \\ ${ }^{1}$ Department of Neurology, National Institute of Mental Health and Neurosciences (NIMHANS), Bangalore, India \\ ${ }^{2}$ Department of Psychological and Brain Research, Johns Hopkins University, Baltimore, USA \\ ${ }^{3}$ Department of Pediatric Surgery, Government Sree Avittom Tirunal Institute of Child Health, Kerala, India \\ ${ }^{4}$ Department of Neurochemistry, National Institute of Mental Health and Neurosciences, Bengaluru, India \\ ${ }^{5}$ Department of Psychiatry, National Institute of Mental Health and Neurosciences, Bengaluru, India
}

\begin{abstract}
Introduction: The word ectoderm is derived from Greek "ektos" which means "outside" and "derma", meaning "skin". This differentiates in to the nervous system, teeth, skin, sweat glands, hair and nails. As these structures develop at almost the same time, diseases affecting the nervous system share changes in one or more of these sites, which serve as an easy marker for diagnosis.

Patient and methods: Patients with various neurological illnesses seen by the authors in the last five years were evaluated for ectodermal markers. They all underwent appropriate investigations to establish the diagnosis.

Observation: Total numbers of patients were 955. Male to female ratio was 1.5:1. Mean age was10 \pm 4.5 years. Out of these patients, clues from general examination helped in either giving a specific diagnosis or helped in formulating differential diagnosis in 78 (8.2\%) patients. The conditions belonged to degenerative, immunological, metabolic, infective, inflammatory and toxic causes.

Conclusion: Ectodermal organs serve as a window to neurological diagnosis in about $8.2 \%$ children presenting with Global Developmental Delay (GDD) and this clinical clue narrows down the differential diagnosis in children in the first decade of their life.
\end{abstract}

Keywords: Skin, hair, nail, ectoderm, neurological diagnosis.

Accepted on September 01, 2018

\section{Introduction}

Both the skin and nervous system develop from the ectoderm, which could be one of the reasons that many diseases of the skin show their counterpart in the nervous system and vice versa. This information when carefully applied will bring down differential diagnosis in disease situations to a relatively limited list. Generally, these disorders are categorized as Phakomatoses, immunological, infectious and genetic diseases [1]. The diseases for which the markers can be used include: Neurocutaneous disorders, disorders associated with impaired immunity, stroke, neuropathy, meningitis or meningoencephalitis, vesicular lesions, ecchymoses, nonpalpable purpura, and petechiae, café-au-lait spots, amyloidosis, rheumatoid arthritis, cutaneous vasculitis, photosensitivity, and melanoma [2]. Skin forms 15 to $20 \%$ of body weight and is the largest human organ. Hair is a skin appendage which undergoes cyclical change and develops in the $3^{\text {rd }}$ month of gestation. Nails are also modified epidermis and start developing from the $10^{\text {th }}$ week of gestation. Therefore, skin and its appendages are capable of carrying markers of a large group of systemic diseases [3].

Immune compromised states carry infective and non-infective markers [4]. Serious diseases like strokes can also have markers like Amyloidosis VII, Cutis laxa, Behçet's disease, Erythema nodosum, genital and oral aphthous ulcers, cerebral cavernous malformations, etc. Skin changes of Diabetes mellitus, Endocarditis, Fabry's disease and hereditary hemorrhagic telangiectasia show telangiectasias, xanthomas, xanthelasma, scleroderma and systemic lupus erythematosus associated manifestations [5].
Disorders associated with peripheral neuropathy are as follows: seborrheic dermatitis in HIV, malar rash in primary Amyloidosis, alopecia in Purpura, bullae in Rheumatoid Arthritis; Mees' lines in arsenic toxicity; angiokeratomas, and hyperkeratosis in Fabry's Disease; bronze pigmentation in Haemochromatosis, jaundice, ichthyosis and panniculitis in Lupus, leonine facies, erythema nodosum and Lucio phenomenon due to arteritis of skin in Hansens disease, vitiligo, nevi sebaceous of Jadassohn in Linear Nevus Sebaceous Syndrome and velvety skin of pellagra, and rhagades in nutritional deficiencies, hyperpigmentation, oedema, hypertrichosis and Raynaud's phenomenon in POEMS syndrome, photosensitivity, short wooly hair, black knuckle pigmentation, scleroderma-like skin, graying hair and baldness, leg ulcers etc.in Vitamin B12 deficiency [6-8]. A wide spectrum of skin changes is seen in both infective and aseptic meningitis [9]. Patients with Neurofibromatosis type 1 present with café-au-lait spots, freckling, plexiform neurofibromatosis, and blue-red macules [10,11]. But these features are less common with type 2 Neurofibromatosis [12]. As skin, hair, nails, teeth and the nervous system share a common embryology of the ectodermal origin; diseases of early development affect these structures together and serve as a useful diagnostic tool.

\section{Patients and Methods}

Patients of pediatric age group (In first and second decade of their life) seen at our Centre in last 5 years from March 2012 to March 2017 at National Institute of Mental Health and Neurosciences (NIMHANS), Karnataka state, South India, were 
Citation: Chandra SR, Suresh A, Annapoorni CSV, et al. Skin, hair and nails as pointers to neurocognitive disorders in children: our experience. $J$ Clin Psychiatry Cog Psychol. 2018;2(2):5-10.

reviewed. Patients in whom skin, hair, nails and teeth helped in diagnosis or evaluation were tabulated. The patient's clinical details and bedside markers were identified and diagnosis was tabulated. Written consent taken from all patients.

\section{Results}

Total numbers of patients were 955. Male to female ratio was 1.5:1. Mean age of $10 \pm 4.5$ years. A majority of the patients seen had refractory seizures and Global Developmental Delay as part of post-hypoxic and post-encephalitis sequelae. Among the rest, clues from general examination helped in either giving a specific diagnosis or in formulating differential diagnosis in $78(8.2 \%)$ patients. Skin was of use in the following cases: Neurofibromatosis-14, Tuberous sclerosis-19 $[13,14]$, Sturge Weber syndrome-9 [15], Phenylketonuria-6 [16], Dermatomyositis-2 [17], Adrenoleucodystrophy-4 [18], Mitochondrial cytopathy-4 [19], Marinesco-Sjogren syndrome-1 [20], Sjogren-Larsson syndrome-1 [21], Ethyl malonic aciduria-1 and GM1 gangliosidoses-2. Teeth and nail were of clue in the following: Wilson's disease-2, Tetracycline toxicity-1, Congenital syphilis-1, Arsenic toxicity -2 and Lead toxicity-1. Hair was of diagnosis in the following cases: Phenylketonuria-6, Biotinidase deficiency-3, Mucopolysaccharidoses-2, Giant axonal neuropathy-1 and Menkes kinky hair syndrome-1(Figures 1-3).

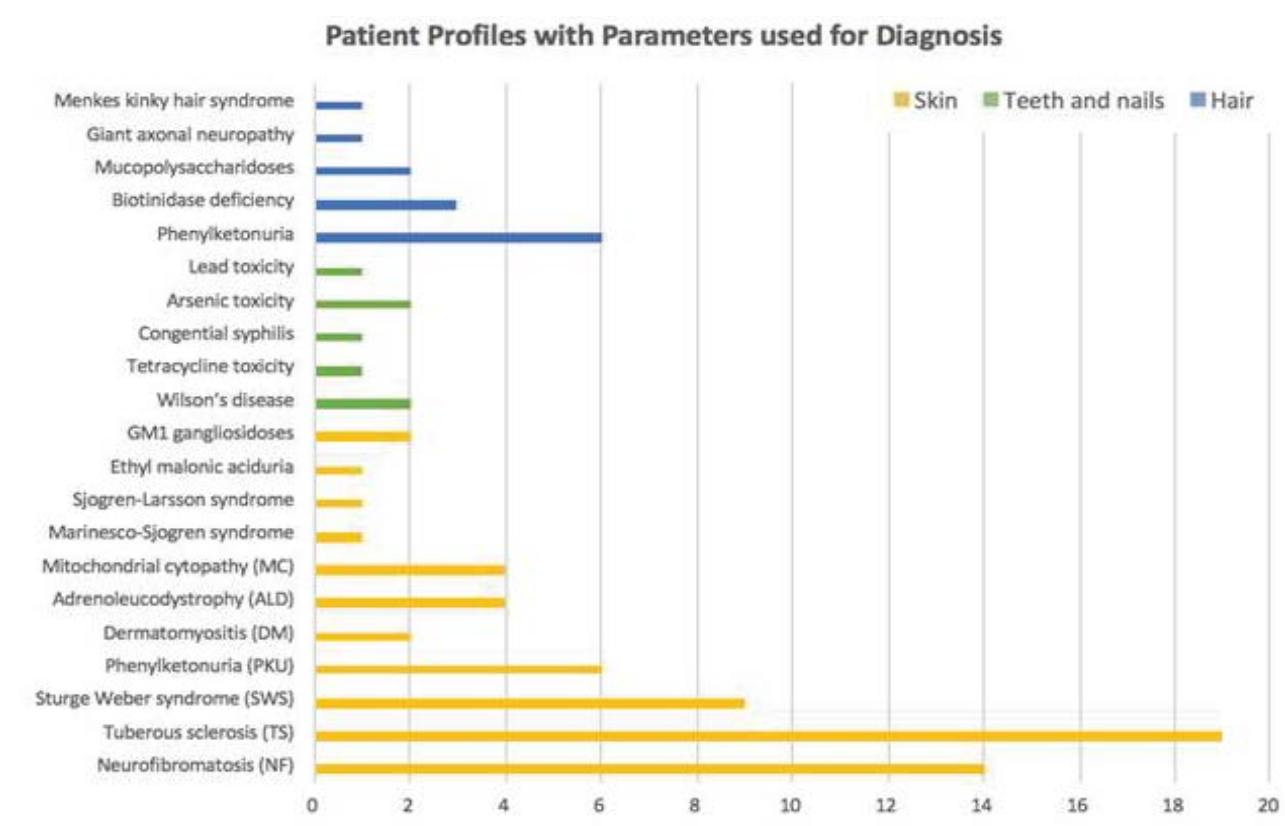

Figure 1: Profile of Patients.

\begin{tabular}{|c|c|c|}
\hline Diagnosis & Patients & Type \\
\hline Neurofibromatosis (NF) & 14 & Skin \\
\hline Tuberous sclerosis (TS) & 19 & Skin \\
\hline Sturge Weber syndrome (SWS & 9 & Skin \\
\hline Phenylketonuria (PKU) & 6 & Skin \\
\hline Dermatomyositis (DM) & 2 & Skin \\
\hline Adrenoleucodystrophy (ALD) & 4 & Skin \\
\hline Mitochondrial cytopathy (MC) & 4 & Skin \\
\hline Marinesco-Sjogren syndrome & 1 & Skin \\
\hline Sjogren-Larsson syndrome & 1 & Skin \\
\hline Ethyl malonic aciduria & 1 & Skin \\
\hline GM1 gangliosidoses & 2 & Skin \\
\hline Wilson's disease & 2 & Teeth and nails \\
\hline Tetracycline toxicity & 1 & Teeth and nails \\
\hline Congential syphilis & 1 & Teeth and nails \\
\hline Arsenic toxicity & 2 & Teeth and nails \\
\hline Lead toxicity & 1 & Teeth and nails \\
\hline Phenylketonuria & 6 & Hair \\
\hline Biotinidase deficiency & 3 & Hair \\
\hline Mucopolysaccharidoses & 2 & Hair \\
\hline Giant axonal neuropathy & 1 & Hair \\
\hline Menkes kinky hair syndrome & 1 & Hair \\
\hline Total: & 77 & \\
\hline Key & Tally & \\
\hline Skin & 63 & \\
\hline Teeth and nails & 7 & \\
\hline Hair & 13 & \\
\hline
\end{tabular}

Figure 2: Distribution of skin, hair, nails changes. 
USE OF SKIN, TEETH AND NAILS, AND HAIR IN DIAGNOSIS

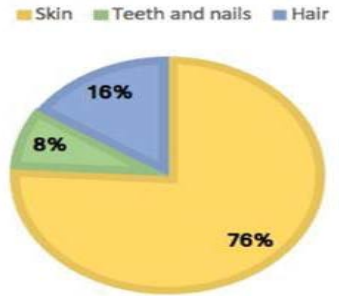

Figure 3: Percentage of utility in ectodermal appendages.

\section{Discussion}

In $8.2 \%$ of children, a reasonable diagnosis could be made with clinical examination as the only tool. The neurodegenerative disorder identified most commonly is tuberous sclerosis which manifested with ash leaf macule, shagareen patch and adenoma sebaceum (Figure 4). Hypo pigmented leafy macules, epilepsy, mental subnormality, hyperactivity and cardiac rhabdomyoma can also be seen. This is due to hyperactivity of the mechanistic target of rapamycin complex 1 (mTORC1), which constitutes the molecular basis and thus, mTORClinhibitors (Everolimus) can be tried. As it is dominantly inherited, prenatal testing should be advised for subsequent pregnancies. NF 1 and 2 and Sturge Weber syndrome are the next commonest identified conditions showing the angioma (Figure 5). Marinesco-Sjogren syndrome is caused by mutations of the SIL1 gene which has the following features: ataxia, cataracts, muscle weakness, hypergonadotrophic hypogonadism, dry skin, short stature and cerebellar atrophy [22] (Figure 6). Sjogren-Larsson syndrome patients have dry scaly skin, spasticity, retinal crystals and leukoencephalopathy [23] (Figure 7). Adrenoleukodystrophy (ALD) is an X-linked recessive disease which presents with dark pigmentation, blindness, seizures and spasticity and, sometimes, neuropathy. Imaging shows periventricular changes around the occipital horn with enhancing margins. There is mutation in the gene that makes ALDP causing abnormally high levels of Very Long Chain Fatty acids (VLCFAs) [24,25] (Figures 8-10). Mucopolysaccharidosis is a group of disorders due to defects in lysosomal enzymes involved in the breakdown of muycopolysaccharides called as glycosaminoglycans. Patients present with mental retardation, coarse skin, bushy eyebrows, flat nose, corneal clouding, visceral and skeletal changes [19] (Figure 11). Wilson's disease is caused by abnormal deposition of copper in the liver and brain due to copper transport by the hepatic lysosomes [26]. This is an autosomal recessive disease that is easily treatable if diagnosed early. It occurs due to impaired function of P-type adenosine triphosphatase (ATPase), encoded by the ATP7B gene located on chromosome $13 q 14$ and consisting of 21 exons. Darkening of skin, brown teeth and neurological symptoms involving cerebellum, basal ganglia as well as other parts of neuraxis at different stages is seen [27] (Figure 12). Dermatomyositis is an immunemediated disease affecting the skin and muscles (Figure 13). This is associated with Anti-Mi-2AB and shows heliotrope rash, Gottron's papules, the V-neck sign, the shawl sign, cuticular overgrowth, and photosensitivity. Anti-transcriptional intermediary factor 1 gamma (TIF $1 \gamma)$-positive patients show photoerythema, "dusky red face," while skin ulcerations, palmar papules (inverse Gottron), diffuse hair loss, panniculitis, and oral pain and/or ulcers are sometimes associated with antimelanoma differentiation-associated gene 5 product (MDA5) antibody [28]. Patients with giant axonal neuropathy show the typical thick curled hair, neuropathy and mental retardation [29] (Figure 14). Phenyl ketonuria patients present with pale colour of skin, hair and even burnt skin appearance, in addition to mental retardation and seizures [30] (Figure 15). Biotinidase deficiency shows reduced scalp hair with hypotonia, ataxia and developmental delay, vision problems, hearing loss, skin rash and greying of hair [31] (Figures $16 \& 17$ ). Toxins like arsenic show rain drop pigmentation [32] and lead shows Mee's' lines [33] (Figures $18 \&$ 19). These observations are interesting as the diseases belong to a wide spectrum of conditions with unique markers, making diagnosis relatively easy. Here we have discussed a group of disorders belonging to genetic, metabolic, toxic as well as degenerative pathology involving various structures of the nervous system with unique clues pointing to specific diagnosis in derivatives of the ectoderm like skin, hair and nails which helps in focused investigation.
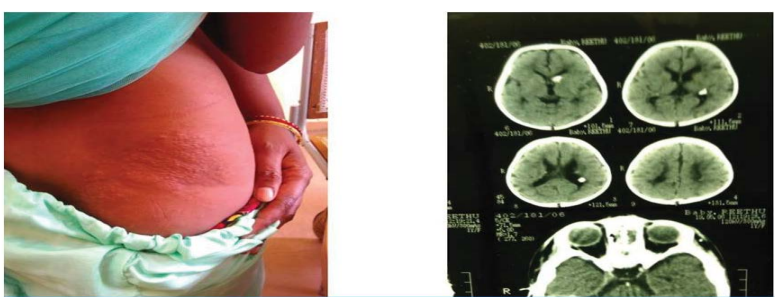

Figure 4: Shagreen patch tuberous sclerosis.

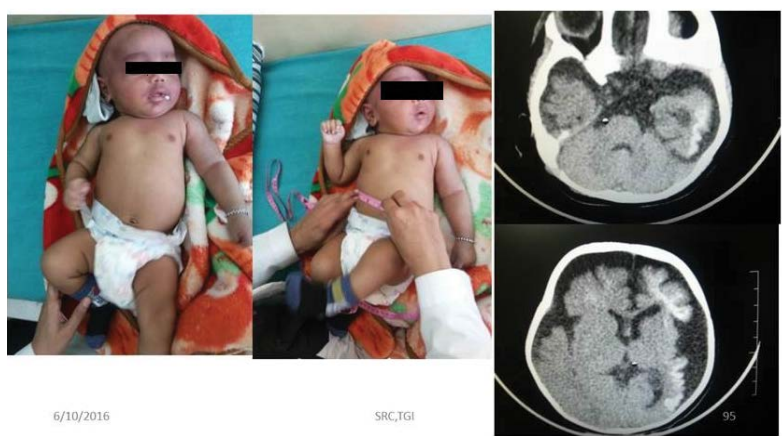

Figure 5: Angioma Klippel Trenany syndrome.
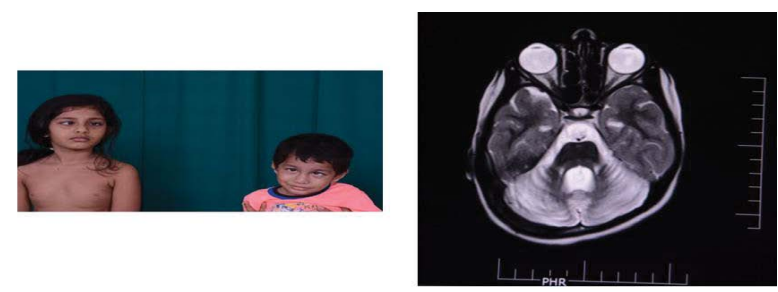

Figure 6: Marinesco sjogren syndrome.

\section{Significance}

Accurate diagnosis of problems in children is very essential as they are at a stage of development and serious sequelae will result if not diagnosed and treated early. Clinical examination offers easy clues to narrow down the differential diagnosis by 
Citation: Chandra SR, Suresh A, Annapoorni CSV, et al. Skin, hair and nails as pointers to neurocognitive disorders in children: our experience. J Clin Psychiatry Cog Psychol. 2018;2(2):5-10.

helping us to focus on the most important conditions. Moreover, confirmation of diagnosis is mandatory to provide prognosis to parents, as well as to advise them regarding subsequent pregnancy. In resource-constrained settings, simple bedside clues are very useful in reducing the cost of diagnostic tools.

- $11 / 2 \mathrm{yr}$

- Global delay

- Lower limb spasticity

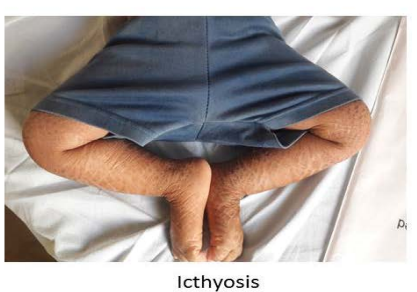

Figure 7: Sjogren larsen syndrome.

- $13 \mathrm{yr}, \mathrm{M}$

- Intellectual disability

- Progressive spasticity of lower limbs
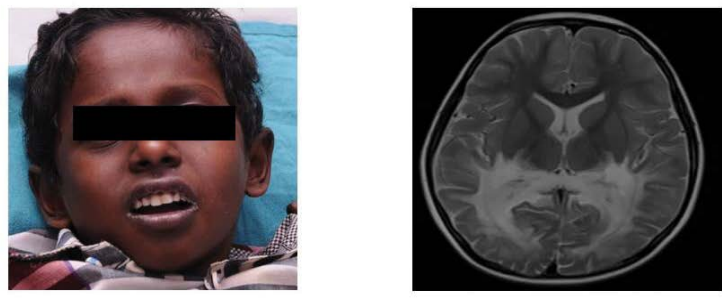

Figure 8: Adreno leukodystrophy.

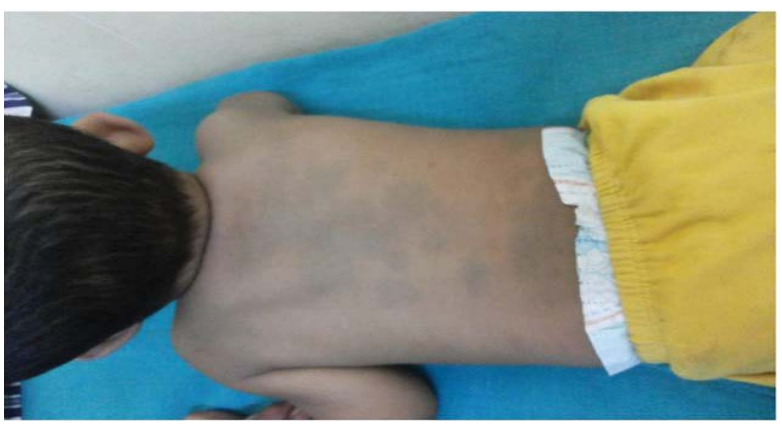

Figure 9: GM1 gangliosidosis.
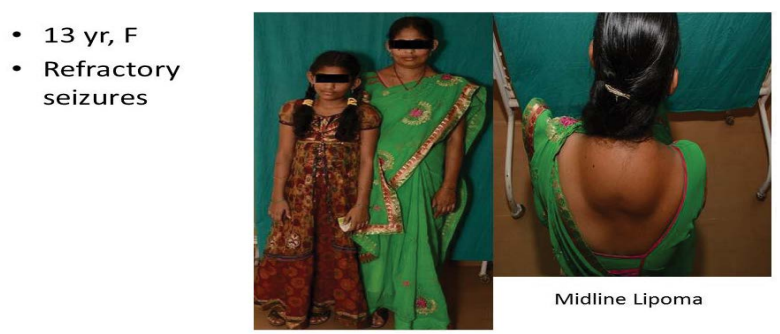

Figure 10: MERRF
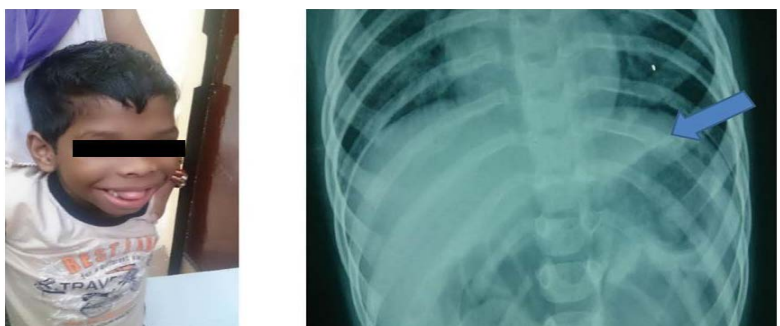

Figure 11: MPS
- $11 \mathrm{yr}, \mathrm{M}$

- Intellectual disability

- Progressive dystonia

- Dysarthria

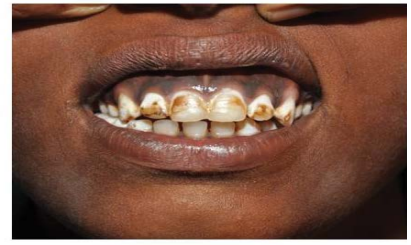

Brown Teeth

Figure 12: Wilsons disease brown teeth.

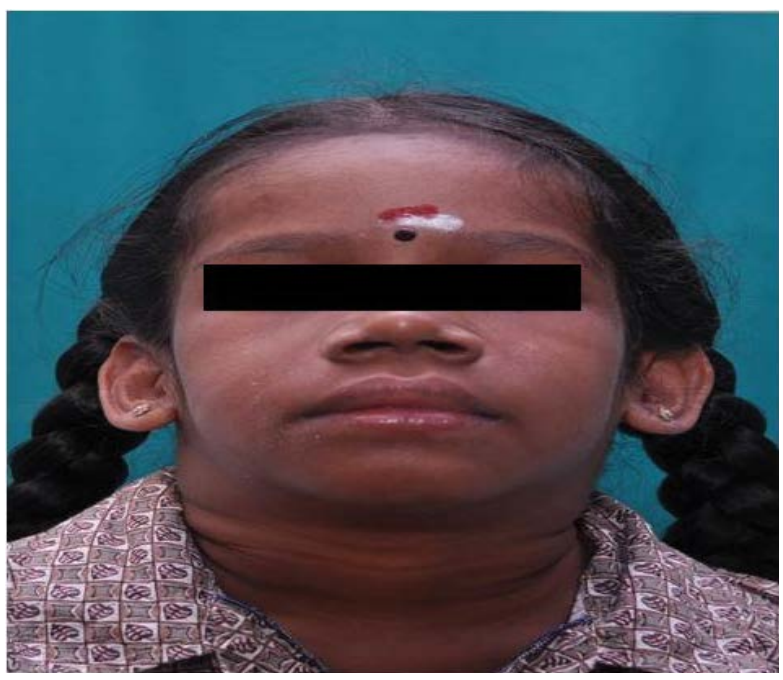

Figure 13: Dermatomyositis.

- 10 yrs, $\mathrm{F}$

- Intellectual disability

- Mild ataxia

- Bipyramidal signs

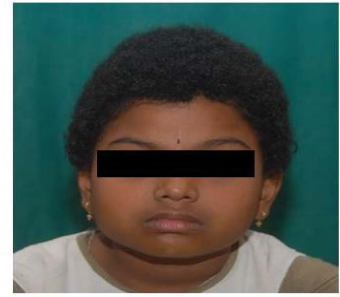

Tight curled hair

Figure 14: Giant axonal neuropathy.
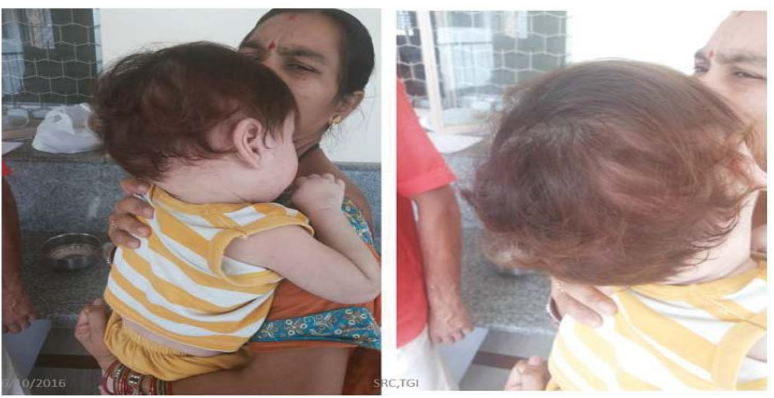

Figure 15: Phenyl Ketonuria.

- 10 months

- Global delay

- Seizures

- Hypotonia

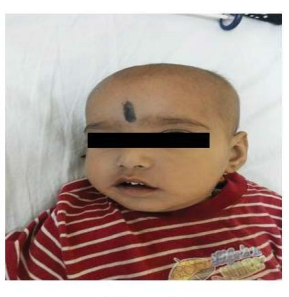

Alopecia

Figure 16: Biotinidase deficiency 
- 6 months

- global developmental delay

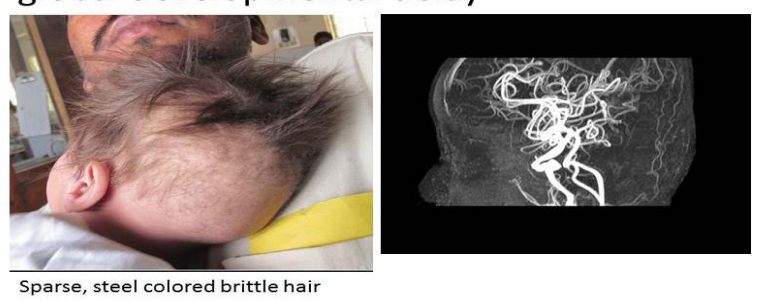

Figure 17: Menkes Kinky hair syndrome.
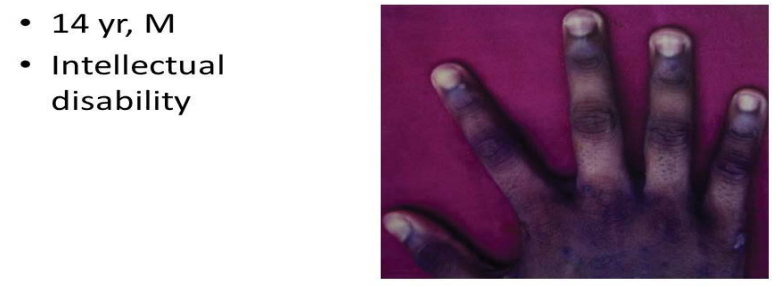

Rain drop pigmentation
Mees lines

Figure 18: Rain drop pigmentation arsenic poisoning.
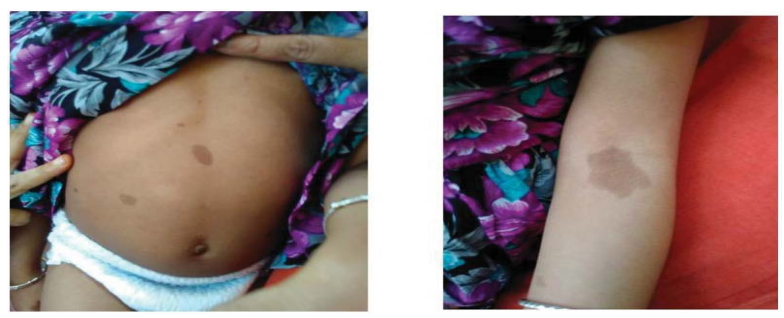

Figure 19: Cafe' au lait spot NF1.

\section{Conclusions}

Skin, hair and nails should always be examined in all children with neurological illnesses as this observational study reveals that $8.2 \%$ of children with various serious neurological disorders show simple clues in these organs. Most of the children with neurological disorders become clubbed under an umbrella term of global or restricted developmental delays. As specific changes in the skin, hair or nail points to specific disorders causing neurological abnormalities, knowledge into these factors serves as an easy clinical pointer for focused diagnosis of the cause for global or restricted neurodevelopmental problems.

\section{Acknowledgement}

I acknowledge with gratitude The National Institute of mental Health and Neurosciences, Bangalore and Karnataka state, South India for the encouragement

\section{REFERENCES}

1. Hurko O, Provost TT. Neurology and the skin. J Neurol Neurosurg Psychiatry. 1999:66(4);417-30.

2. Sontheimer RD, Provost TT. Cutaneous manifestations of rheumatic disease. Baltimore: Williams and Wilkins. 1996.

3. Bailey BJ, Briars GL. Estimating the surface area of the human body. Stat Med. 1996:15(13);1325-32.
4. Lynch $\mathrm{MH}$, O'Guin WM, Hardy $\mathrm{C}$ et, al. Acidic and basic hair/nail ("hard") keratins: Their colocalization in upper cortical and cuticle cells of the human hair follicle and their relationship to" soft" keratins. J Cell Biol. 1986:103(6);2593-606.

5. Lipsker D. Cutaneous Manifestations of Internal Diseases. Clinical Examination and Differential Diagnosis of Skin Lesions, Paris. 2013.

6. Feitosa MF, Borecki I, Krieger $\mathrm{H}$, et al. The genetic epidemiology of leprosy in a Brazilian population. Am J Hum Genet. 1995:56(5);1179-85.

7. Noordeen SK, Bravo LL, Sundaresan TK. Estimated number of leprosy cases in the world. Bull World Health Organ. 1992:70(1);7-10.

8. Mosher DB. Disorders of Pigmentation. Dermatology in general medicine.

9. Farah S, Al-Shubaili A, Montaser A, et al. Behçet's syndrome: A report of 41 patients with emphasis on neurological manifestations. J Neurol Neurosurg Psychiatry. 1998:64(3);382-4.

10. Fenichel GM. Clinical pediatric neurology: A signs and symptoms approach. Elsevier Health Sciences. 2009.

11. Little H, Kamat D, Sivaswamy L. Common neurocutaneous syndromes. Pediatr Ann. 2015:44(11);496-504.

12. Westerhof W, Beemer FA, Cormane RH, et al. Hereditary congenital hypopigmented and hyperpigmented macules. Arch Dermatol. 1978:114(6);931-6.

13. Northrup H, Koenig MK, Pearson DA, et al. Tuberous Sclerosis Complex-Somatic Mosaicism. 2015.

14. Riccardi VM. Von Recklinghausen neurofibromatosis. N Engl J Med. 1981:305(27);1617-27.

15. Thomas-Sohl KA, Vaslow DF, Maria BL. Sturge-Weber syndrome: A review. Pediatr Neurol. 2004:30(5);303-10.

16. Crome L, Pare CM. Phenylketonuria: A review and a report of the pathological findings in four cases. Br J Psychiatry. 1960:106(444);862-83.

17. Ang P, Sugeng MW, Chua SH. Classical and amyopathic dermatomyositis seen at the National Skin Centre of Singapore: A 3-year retrospective review of their clinical characteristics and association with malignancy. Ann Acad Med Singapore. 2000:29(2);219-23.

18. Moser HW, Mahmood A, Raymond GV. X-linked adrenoleukodystrophy. Nat Rev Neurol. 2007:3(3);140-51.

19. Bodemer C, Rötig A, Rustin P, et al. Hair and skin disorders as signs of mitochondrial disease. Pediatrics. 1999:103(2);428-33.

20. Yvonneau M, Dupouy F. Marinesco-Sjögren syndrome. L'Encephale. 1977:3(2);165-72.

21. Rizzo WB. Sjögren-Larsson syndrome. Semin dermatol. 1993:12(3);210-18.

22. Kashimada A, Hasegawa S, Isagai $\mathrm{T}$, et al. Targeting the enhanced ER stress response in Marinesco-Sjögren syndrome. J Neurol Sci. 2018:385;49-56. 
23. Henkin RI, Talal N, Larson AL, et al. Abnormalities of taste and smell in Sjogren's syndrome. Ann Intern Med. 1972:76(3);375-83.

24. Schaumburg HH, Powers JM, Raine CS, et al. Adrenoleukodystrophy: A clinical and pathological study of 17 cases. Arch Neurol. 1975:32(9);577-91.

25. Natarajan A, Christopher R, Netravathi M, et al. Liquid chromatography-tandem mass spectrometry method for estimation of a panel of lysophosphatidylcholines in dried blood spots for screening of X-linked adrenoleukodystrophy. Clin Chim Acta. 2018:485;305-10.

26. Esko JD, Kimata K, Lindahl U. Proteoglycans and sulfated glycosaminoglycans. Essentials of Glycobiology. 2009.

27. Taly AB, Meenakshi-Sundaram S, Sinha S, et al. Wilson disease: Description of 282 patients evaluated over 3 decades. Medicine. 2007:86(2);112-21.
28. Muro Y, Sugiura K, Akiyama M. Cutaneous manifestations in dermatomyositis: Key clinical and serological featuresA comprehensive review. Clin Rev Allergy Immunol. 2016:51(3);293-302.

29. Crete R, Jorgensen SA, Towbin AJ, et al. Giant axonal neuropathy. Appl Radiol. 2018:47(5);40-1.

30. Chandra SR, Christopher R, Daryappa MM, et al. Phenylketonuria: Our experience in nine years at a tertiarylevel referral institute. J Pediatr Neurosci. 2018:13(1);62-70.

31. Sakamoto O, Narisawa K. Biotinidase deficiency (late-onset multiple carboxylase deficiency). Ryoikibetsushokogunshirizu. 1998.

32. Nickson R, McArthur J, Burgess W, et al. Arsenic poisoning of Bangladesh groundwater. Nature. 1998:395(6700);338.

33. Needleman H. Lead poisoning. Annu. Rev. Med. 2004:55:209-22.

\section{*Correspondence to:}

S.R. Chandra

Department of Neurology, National Institute of Mental Health and Neurosciences (NIMHANS), Bangalore, India

E-mail -drchandrasasi@yahoo.com 